\title{
Leonid electrophonic bursters
}

\author{
M. Beech ${ }^{1}$ and L. Foschini ${ }^{2}$ \\ 1 Campion College and Department of Physics, The University of Regina, Regina, Saskatchewan, \\ Canada S4S 0A2 \\ 2 Institute TeSRE - CNR, Via Gobetti 101, 40129 Bologna, Italy \\ e-mail: foschini@tesre.bo.cnr.it
}

Received 4 October 2000 / Accepted 27 November 2000

\begin{abstract}
We investigate the conditions under which Leonid meteoroids might generate short duration (burster) electrophonic sounds. A "first order" theory is employed to estimate the approximate electron number density in the meteoroid ablation column as a function of time. Using the threshold conditions discussed in an earlier communication (Beech \& Foschini 1999) we find that Leonid meteoroids more massive than about $0.1 \mathrm{~kg}$ can potentially generate short duration electrophonic bursters.
\end{abstract}

Key words. meteors, meteoroids

\section{Introduction}

The existence, or not of electrophonic sounds has long been a contentious issue within meteor studies. The essential mystery of what are now called electrophonic sounds is that observers report hearing "crackling" or "rushing" and sometimes "popping" sounds at the same time as seeing a fireball. The sounds often mimic the behaviour of the meteor, rising and falling as a meteor fragments and flares. While such sounds have been reported throughout antiquity, there has until very recently been little experimental investigation of the phenomenon in the field (Beech et al. 1995; Beech \& Nikolova 1999; Garaj et al. 1999). For all the contention, however, Keay $(1980,1993)$ and later on Bronshten (1983), have presented a clearly described and testable model for the generation of enduring electrophonic sounds. In addition, Beech \& Foschini (1999) have presented the outlines of a model for the origin of the short duration, so called, burster electrophonic sounds. Generally speaking enduring electrophonic sounds may be associated with the appearance of slow, sporadic fireballs (Keay \& Ceplecha 1994), but electrophonic bursters have reportedly been heard during Leonid, and Lyrid outbursts and annual Perseid meteor shower displays.

In this paper, we are specifically concerned with the Leonid meteoroid stream. In an earlier study, Beech (1998) found that enduring electrophonic sounds might be produced from (of order of) metre-sized Leonid meteoroids. Clearly, such large objects are not going to be abundant in the Leonid stream at any one time, but it has been

Send offprint requests to: M. Beech,

e-mail: Martin.Beech@uregina.ca suggested by Beech \& Nikolova (2000) that they might be deposited into the stream during mantle ejection events associated with the aging of comet $55 \mathrm{P} /$ Tempel-Tuttle. Reports gathered by Olmsted during the 1833 Leonid storm indicate that enduring electrophonic sounds, as well as electrophonic bursters, were heard and this is indicative of the presence of at least a few large Leonid meteoroids. Of more recent note, however, electrophonic bursters have reportedly been heard during both the 1998 (Darren Talbot, personal communication) and 1999 (Drummond et al. 2000; Beatty 2000) Leonid outbursts.

\section{The electrophonic burster model}

The various catalogues that have been compiled on electrophonic sounds indicate that about $10 \%$ of the reports fall into what we call the "burster" category. The bursters are characterized by being short in duration (typically a second or less), and they are often described as sounding like "pops", or "vits" and even "sharp clicks". The other key characteristic of the burster events is that they are often, but not always, associated with a single, dramatic break-up event of the ablating meteoroid.

Beech \& Foschini (1999) have suggested that the burster phenomenon can be explained in terms of a shock propagating within a meteor's plasma column. Within the framework of this model, it is argued, that the rapid movement of the electrons, with respect to the much more massive and slower moving ions, generates a sizable space charge (see e.g., Zel'dovich \& Raizer 1967). A transient electrical pulse is generated in response to the development of the space charge, and provided the resultant 
electrical field strength variations are large enough it is suggested, following Keay (1980), that they might trigger the generation of audible sounds through an observer localized transduction process. The shock wave is produced, Beech \& Foschini (1999) suggest, during the catastrophic break-up of the parent meteoroid.

Under ideal conditions the electric field generated would be symmetric, propagating equally in all directions. However, as is more likely, the presence of fluid dynamic instabilities will lead to preferential propagation in certain directions. Specifically, since the meteor plasma can be thought of as a fluid with a higher density than the surrounding atmosphere, both Rayleigh-Taylor and Kelvin-Helmholtz instabilities might develop. In the possible presence of these instabilities, any shock front will soon become distorted. One may therefore envision the situation in which the electric field is either focused or defocused in random directions by the growing filaments and small scale perturbations in the shock. Gull (1975) has described the complex instability modification of a shock front within the context of a stellar supernovae model. The same processes are likely to occur in airburst explosions, with obvious changes in energy scale.

In addition to the presence or not of appropriate transduction material, this instability feature may explain why electrophonic sounds often appear to be highly localized. Experiments conducted by Keay \& Ostwald (1991) suggest that an electric field strength of at least $160 \mathrm{~V} / \mathrm{m}$ is required to generate electrophonic sounds. Beech \& Foschini (1999) find that such a threshold electric field can be generated provided the electron number density within the plasma exceeds $n_{\mathrm{e}} \approx 410^{18} \mathrm{~m}^{-3}$.

\section{The ionization coefficient}

In this section we shall attempt to constrain the critical mass above which a Leonid meteoroid might produce an electrophonic burster. We shall employ order of magnitude arguments at this stage, leaving refinements of the physics to future detailed numerical modeling. The great complexity of the meteoroid interaction with the Earth's atmosphere is discussed, for example, in Ceplecha et al. (1998) and Foschini (1999). The variation in the electron line density, $q$, produced by an ablating meteoroid can be described as (Kaiser 1953; Hughes 1978):

$q=-\frac{\beta}{\mu V} \frac{\mathrm{d} m}{\mathrm{~d} t}$

where $V$ is the velocity, $\beta$ is the ionization coefficient, $\mu$ is the mean atomic mass of meteoroid atoms, and $\mathrm{d} m / \mathrm{d} t$ is the mass ablation rate. The mass loss rate can be derived by solving the standard single-body equations of meteoroid ablation (see, e.g. Öpik 1958; McKinley 1961).

To first order, the electron line density may be converted to an electron number density $n_{\mathrm{e}}$ via the relation:

$q=\pi r_{\mathrm{i}}^{2} n_{\mathrm{e}}$ where $r_{\mathrm{i}}$ is the initial train radius. Equation (2) is the limiting initial case of the diffusion equation when $t \approx 0$ (see e.g., Mckinley 1961). Provided one can establish analytically reasonable expressions for the ionization coefficient, $\beta$, and the initial train radius, $r_{\mathrm{i}}$, then Eqs. (1) and (2) may be solved for numerically. In this manner, the characteristics of the Leonid meteoroid just capable of producing an electron number density of, say, $10^{19} \mathrm{~m}^{-3}$ (this places $n_{\mathrm{e}}$ above the burster threshold condition derived by Beech \& Foschini 1999) can be determined.

The core of the problem is the determination of the ionization coefficient $\beta$. It is, by definition, the ratio of the number of free electrons produced to the number of meteoroid atoms vaporized. The ionization coefficient is strongly dependent upon the relative speed with which the meteoroid's ablated atoms and atmosphere molecules collide. Typically the ionization coefficient is expressed as a power law in the velocity

$\beta=\beta_{0} \cdot V^{n}$

where $\beta_{0}$ and $n$ are coefficients to be calculated.

Massey \& Sida (1955) and later on Sida (1969) studied the collision processes in meteor trails and argued that $\beta$ can be expressed as the ratio of the ionizing and momentum-loss cross sections. It is the sensitivity of the ionization cross section to the relative velocity that introduces the velocity dependency in $\beta$. Massey and Sida proposed several different ways of weighting the cross sections, taking into account different factors such as the angle of scattering (see Jones 1997, for a discussion of the inherent problems with the Massey and Sida model). Indeed, even though the definition of $\beta$ is quite straightforward, the complexity of particle dynamics in the meteor plasma complicates the situation greatly, and the correct weighting factors are still a topic of debate (for a recent review on particle dynamics in meteor plasma's see Dressler \& Murad 2000).

Bronshten (1983) presents a detailed analysis of the empirical and theoretical calculations of $\beta$ and finds that:

$\beta=5.4710^{-7} \cdot V^{3.42}$.

where the velocity $V$ is expressed in $\mathrm{km} \mathrm{s}^{-1}$. Jones (1997) has recently provided a thorough review of the methods by which $\beta$ might be calculated, and finds that for visual meteors with velocities in the range of 30 to $60 \mathrm{~km} \mathrm{~s}^{-1}$.

$\beta=4.9110^{-6} \cdot V^{2.25}$.

In the theory developed by Jones $\beta<1$, while Bronshten considered the situation when $\beta$ is greater than unity. In this later case, secondary ionization is included under the supposition that at very high relative velocities a single meteoroid atom can produce more than one free electron. Within the context of this first order study we choose to use both approximations for the ionization coefficient and present two sets of comparative calculations.

As with the ionization coefficient, the initial train radius is also a difficult term to quantify. Generally speaking it will be of order the atmospheric mean free collision 
Table 1. Critical Leonid meteoroid mass limits for generating electrophonic bursters. Column one indicates which form of the ionization coefficient was employed in the calculations; the second column indicates the meteoroid composition; column three corresponds to the assumed zenith angle of entry; column four indicates the height at which $n_{\mathrm{e}}$ first exceeds the $10^{19} \mathrm{~m}^{-3}$ threshold; column five indicates the approximate visual magnitude of the meteoroid at $h_{\text {crit }}$

\begin{tabular}{llcccc}
\hline Ionization Theory & Composition & $Z[\mathrm{deg}]$. & $m_{\text {crit }}[\mathrm{kg}]$ & $h_{\text {crit }}[\mathrm{km}]$ & $M_{\text {vis }}$ \\
\hline Jones (1997) & type IIIA & 0 & 0.028 & 80.35 & -8.6 \\
& & 20 & 0.031 & 80.60 & -8.7 \\
& & 45 & 0.051 & 81.30 & -8.9 \\
& type IIIB & 0 & 0.12 & 85.80 & -10.4 \\
& & 20 & 0.14 & 85.90 & -10.4 \\
& & 45 & 0.22 & 86.73 & -10.7 \\
\hline Bronshten (1983) & type IIIA & 00 & 0.41 & 87.60 & -11.0 \\
& & 0 & 0.005 & 84.10 & -6.8 \\
& & 40 & 0.006 & 84.25 & -6.8 \\
& & 0.009 & 84.93 & -7.0 \\
& type IIIB & 0 & 0.021 & 89.30 & -8.5 \\
& & 20 & 0.023 & 89.43 & -8.6 \\
& & 45 & 0.039 & 90.20 & -8.8 \\
& & 60 & 0.070 & 91.08 & -9.1 \\
\hline
\end{tabular}

length. Radar measurements of meteor trains have been used to determine the initial train radius as a function of atmospheric height. Baggaley (1970) found, for example, that the initial train radius was some $3 \mathrm{~m}$ at an altitude of $115 \mathrm{~km}$, and some $0.5 \mathrm{~m}$ at $90 \mathrm{~km}$ altitude. These values of the initial train radius are some 2 to 20 times the mean free path lengths at the respective atmospheric heights. Jones (1995) has derived a relationship for the initial train radius as a function of the number density $n_{\mathrm{a}}$ of air molecules. He finds:

$r_{\mathrm{i}}=2.84510^{18} \frac{V^{0.8}}{n_{\mathrm{a}}}$

where $V$ is the velocity measured in $\mathrm{km} \mathrm{s}^{-1}$. The power of 0.8 in the velocity term describes the energy sensitivity of the ionic scattering cross-section.

\section{The critical mass}

Gathering together Eqs. (1) through (6) and solving them in conjunction with the single body ablation equations we may proceed to determine the electron number density as a function of atmospheric height for a given initial mass. The initial velocity for Leonid meteoroids is determined from the observations to be $71 \mathrm{kms}^{-1}$. The choice of an appropriate meteoroid composition is rather problematic, but we shall consider the so-called type IIIA cometary and type IIIB soft cometary ablation parameters (see, Ceplecha et al. 1998). When solving the single body ablation equations we use the density-height profile provided by the MSIS-E-90 Earth atmosphere model.

The critical mass, $m_{\text {crit }}$, that we are looking for determines the mass of the Leonid meteoroid that will just generate a maximum electron number density of $10^{19} \mathrm{~m}^{-3}$ somewhere along its ablation track. Meteoroids more massive than $m_{\text {crit }}$ will produce electron number densities higher than the critical value, and subsequently should such meteoroids catastrophically disintegrate (when $n_{\mathrm{e}}>$ $10^{19} \mathrm{~m}^{-3}$ ) then the burster mechanism described by Beech \& Foschini (1999) may come into play.

The results of our calculations are presented in Table 1. The calculations begin at an atmospheric height of $190 \mathrm{~km}$ and various zenith angles $(Z)$ of meteoroid entry have been assumed. We have used a constant luminous efficiency of $1 \%$ to determine magnitudes.

The data gathered together in Table 1 suggest that the minimum mass for a Leonid meteoroid to produce an electrophonic burster is somewhere in the range of 5 to $400 \mathrm{~g}$ depending upon zenith angle, composition and the velocity sensitivity of the ionization coefficient. The predicted brightness of the burster meteors is in the magnitude range of -7 to -11 .

For a given zenith angle the variation in composition (types IIIA or IIIB) results in about a factor of 4 variation in the critical mass estimate. For a given composition and zenith angle, the uncertainty in the ionization parameters (expressed through the use of Bronsthen's formula and that of Jones) results in a variation of about 6 in the critical mass.

\section{Some comments on observational techniques}

The contentious aspect of the debate surrounding electrophonic sound production has mostly focused on the issue of observational reliability. Eye-witness and anecdotal accounts are of little value, beyond bolstering the cause, in the modern era. What is required is unambiguous instrumental data. Literally, data that can stand-up to theoretical analysis and detailed scrutiny. Beech et al. (1995) have reported on the detection of an unusual VLF transient associated with the disruption of a -10 visual magnitude Perseid. More recently Garaj et al. (1999) have claimed 
an associative link between a series of very short-duration very low frequency radiation (VLF) transients and a magnitude -8 Leonid fireball. An as yet to be published study conducted by Price et al. during the 1999 Leonid storm (see Beatty 2000) also claims to have recorded many meteor - VLF transient coincidences.

It is our basic belief that none of the observational studies published to date can claim to have clearly established a casual linkage between the generation of a characteristic VLF transient signal and the passage of a fireball. We offer this statement in the sense that none of the published papers present instrumental data that unambiguously shows the coincident detection of a meteor and VLF or electrophonic sound transient.

While it is not our intention to be overly critical of honest effort, we would also like to point out that it is not obviously clear that the correct line of experimental attack has always been employed. Firstly, we should ask ourselves what it is that we are actually trying to measure. Electric and magnetic fields behave differently depending on the distance $R$ between the source and the point of measurement (see, for example, Jackson 1975, Chapter 9). Indeed, we can distinguish three characteristic zones: the near field, the far field, and the intermediate zone. Comparing the distance $R$ to the source with the wavelength $\lambda$, we have

- near field: $R \ll \lambda$;

- far field: $R \gg \lambda$;

- intermediate: $R \approx \lambda$.

Audible frequencies fall in the range of $0.02-4 \mathrm{kHz}$, therefore the minimum wavelength is $75 \mathrm{~km}$, this, we note, is comparable to the meteor heights displayed in Table 1. This is valid if the mundane objects, acting as signal transductors, do not appreciably change the frequency of the incoming electromagnetic radiation. If the transductors do induce a frequency shift, it is possible that the incoming radiation has a higher frequency (and thus, a smaller wavelength).

For more massive meteoroids that penetrate deep into the Earth's atmosphere, we are essentially in the intermediate and near field regions. Only in the far field zone would we actually have a plane transverse electromagnetic wave (and therefore a radiation field). In the burster model discussed above, it is most probable that the intermediate field condition applies and hence the electric and magnetic fields will behave differently. Within this context it may be no coincidence that the VLF transient events reported by Beech et al. (1995) and Garaj et al. (1999) relate to meteors seen close to the horizon (but see below). Indeed, if the source is close to the horizon the distance from the observer can be many hundreds of kilometres. In the longrange case, we have a radiation field and consequently the transmission of energy via the Poynting vector. Before embarking on an experiment to study electrophonic transients a clear decision has to be made with respect to whether it is the electric or the magnetic field component that is to be measured. This decision in turn dictates the choice of antenna to be used in the experiment. The Keay and Bronshten electrophonic model is based upon the relaxation of the geomagnetic field, approximated as a magnetic dipole source, and to study this component one must employ a loop antenna valid for frequencies in the range $50 \mathrm{~Hz}-50 \mathrm{kHz}$. The mechanism outlined by Beech and Foschini for burster electrophonics may be thought of as generating an electric dipole source, and consequently to study this component a vertical wire antenna should be used. If one wishes to test the transduction process directly a well-calibrated detector and microphone system will have to be developed.

In addition to the appropriate selection of an antenna, care should also be directed towards the choice of signal analyzer. The best choice of detector is probably an Electro Magnetic Interference (EMI) analyzer. Such devices are typically sensitive over a wide dynamical range of frequencies. And indeed, we note, that since one is trying to measure implusive or rapidly changing broadband signals an instrument with a small dynamical range will seriously compromise the quality of measurements being made.

The model for electrophonic sound generation described by Keay $(1980,1993)$ makes a clear prediction. That is, the VLF signal generated by the meteoroid interaction with the geomagnetic field will be sustained, possibly observable over an extended period of time, and it will be distinct from background atmospheric sources. While the exact VLF signal characteristics can not be predicted at this stage, it is unlikely that it constitutes a series of very-short duration transients. In this respect, we do not at this stage fully accept that the very short duration VLF transient signals presented by Garaj et al. (1999) in their survey paper represent anything other than background, atmospheric events. They may represent a real meteor related signal, but the evidence it not compelling. The VLF transient signal presented by Beech et al. (1995), on the other hand, has characteristics (signal duration and distinctness from natural atmospheric sources) that make it more believable as an actual meteor related event. Our point here is that unless the meteor generated VLF signal has characteristics that clearly distinguishes it from background sources, then one cannot simply claim that because short-lived VLF transient are observed at the same time that a meteor is seen that the two observations are causally related. It is not clear to us as yet whether the electrophonic bursters are likely to generate a significant VLF signal.

\section{Discussion and conclusions}

Our estimate of the minimum Leonid meteoroid mass for generating electrophonic burster sounds is much smaller than that derived for long enduring electrophonic sounds (of order a hundred grams rather than $800 \mathrm{~kg}$ ). This result, in fact, sits well with the observations since we can be reasonably sure that of order one hundred gram and larger 
mass meteoroids do exist within the Leonid stream (see e.g., Spurny et al. 2000 and Bellot Rubio et al. 2000).

We re-iterate, however, that we do not expect every Leonid fireball of magnitude -7 (and brighter) to generate electrophonic burster sounds. The key point is that the meteoroid has to develop a shock wave at the same time that the electron number density in the plasma column exceeds $10^{19} \mathrm{~m}^{-3}$. In addition, to generate a measurable signal in the VLF range the meteor must be placed at a distance greater than some $75 \mathrm{~km}$ from the observer.

Many of the Leonid fireballs recorded in 1998 were found to began rapid ablation at heights in excess of $120 \mathrm{~km}$. In addition, the average end height of Leonid fireballs was found to be about $85 \mathrm{~km}$ (Spurny et al. 2000). These results certainly indicate that Leonid meteoroids are made of very friable and easily ablated material. Indeed, Spurny et al. (2000) classified all but one of the Leonid fireballs that they recorded as type IIIB, which is typical of the weakest interplanetary bodies. The model outlined above suggests that a meteoroid has to penetrate to an altitude of about 80 to $90 \mathrm{~km}$ before it undergoes catastrophic disruption. On this basis we see no inherent reason why some Leonid fireballs might not produce electrophonic bursters.

One of the key aspects of the enduring electrophonic sound model developed by Keay and Bronshten was the production of very low frequency (VLF) radiation. This radiation is generated through an interaction of the highly ionized and turbulent meteor plasma train with the Earth's magnetic field. It is the transduction of the VLF radiation by objects close to the observer that results in the generation of audible sounds. In contrast, the electrophonic burster model outlined by Beech and Foschini does not specifically predict the generation of any VLF signal. Rather, it predicts the generation of a short-lived transient pulse (or pulses) in electric field strength. The observational campaigns conducted to date have mostly focused upon the detection of VLF radiation transients through monitoring magnetic field variations. Electrophonic bursters, we argue, are more likely to be observed through the generation of electric field transients.

Acknowledgements. We extend our appreciation to the referee, L. Bellot Rubio, for his comments and suggestions. This work has been partially supported by a grant from the Natural Sciences and Engineering Research Council of Canada and partially by MURST Cofinanziamento 2000. This research has made use of NASA's Astrophysics Data System Abstract Service.

\section{References}

Baggaley, W. J. 1970, MNRAS, 147, 231

Beatty, J. K. 2000, Sky Telesc., 99(6), 45

Beech, M. 1998, AJ, 116, 499

Beech, M., Brown, P., \& Jones, J. 1995, Earth, Moon Planets, 68, 181

Beech, M., \& Foschini, L. 1999, A\&A, 345, L27

Beech, M., \& Nikolova, S. 1999, Meteorit. Planet. Sci., 34, 849

Beech, M., \& Nikolova, S. 2000, Planet. Space Sci., in press

Bellot, Rubio, L. R., Ortiz, J. L., \& Sada, P. V. 2000, ApJ, 542, L65

Bronshten, V. A. 1983, Phys. Meteoric Phenomena (D. Riedel Co. Dordrecht)

Ceplecha, Z., Borovička, J., Elford, W. G., et al. 1998, Space Sci. Rev., 84, 327

Dressler, R. A., \& Murad, E. 2000, in Chemical dynamics in extreme environments, ed. R. A. Dressler, Advances Series in Physical Chemistry, vol. 11 (World Scientific, Singapore), in press

Drummond, J., Gardner, C., \& Kelly, M. 2000, Sky Telesc., 99(6), 48

Foschini, L. 1999, A\&A, 341, 634

Garaj, S., Vinkovic, D., Zgrablic, G., et al. 1999, Fizika A (Zagreb), 8, 91

Gull, S. F. 1975, MNRAS, 171, 263

Hughes, D. W. 1978, in Cosmic Dust, ed. J. A. M. McDonnell (Wiley, Chichester), 167

Jackson, J. D. 1975, Classical Electrodynamics (Wiley, New York)

Jones, W. 1995, MNRAS, 275, 812

Jones, W. 1997, MNRAS, 288, 995

Kaiser, T. R. 1953, Philos. Mag. Suppl., 2, 495

Keay, C. S. L. 1980, Science, 210, 11

Keay, C. S. L. 1993, J. Sci. Explor., 7, 337

Keay, C. S. L., \& Ceplecha, Z. 1994, J. Geophys. Res., 99, 13163

Keay, C. S. L., \& Ostwald, P. M. 1991, J. Acoust. Soc. Am., 89, 1823.

Massey, H. S. W., \& Sida, D. W. 1955, Philos. Mag., 46, 190

McKinley, D. W. R. 1961, Meteor Science and Engineering (McGraw Hill, New York)

Öpik, E. J. 1958, Physics of Meteor Flight in the Atmosphere (Interscience, New York)

Sida, D. W. 1969, MNRAS, 143, 37

Spurny, P., Betlem, H., Leven, J. V., et al. 2000, Meteorit. Planet. Sci., 35, 243

Zel'dovich, Y. B., \& Raizer, Y. P. 1967, Physics of Shock Waves and High Temperature Hydrodynamic Phenomena, vol. II (Academic Press, New York) 